\title{
Unusual Presentation of Polyarteritis Nodosa
}

\author{
M M Uddin ${ }^{1}$, M L L Chowdhury ${ }^{2}$, A Yasmin ${ }^{3}$
}

\begin{abstract}
Polyarteritis Nodosa (PAN) is a multisystem, necrotizing vasculitis of small and medium-sized muscular arteries. Sometimes PAN is detected at cholecystectomy or appendectomy in the absence of other disease manifestations. In such cases, surgical removal of the involved organ may be curative. Here we presented a case of poly arteritis nodosa, the process of diagnosis and explained the pathophysiology of the disorder and currently available treatments.
\end{abstract}

\section{Introduction}

PAN was described in 1866 by Kussmaul and Maier. It is a multisystem, necrotizing vasculitis of small and medium-sized muscular arteries in which involvement of the renal and visceral arteries is characteristic. PAN does not involve pulmonary arteries, although bronchial vessels may be involved. Two additional features that distinguish PAN from other forms of systemic vasculitis are confinement of the disease to the arterial rather than the venous circulation, and the absence of granulomatous inflammation. Nonspecific signs and symptoms are the hallmarks of PAN. The gastrointestinal manifestations of PAN occur in approximately half of all patients and are among the most challenging symptoms to diagnose correctly because of their nonspecific nature. Postprandial abdominal pain ("intestinal angina") is common.

\section{Case history}

Popy, 22 yrs old single female admitted with the compliants of abdominal pain, anorexia and nausea since last 2 months. She also complained of melaena since last 10 days. She was completely well 3 yrs back. She presented to the ENT doctor with the complaints of nasal blockage and per nasal bleeding and headache. For that she under went septoplasty in 2007. After that she visited the local doctor for headache; where she was found to have hypertension and impaired renal function. She also had the history of sudden onset of right sided hemiparesis which lasted for 6 months and then resolved completely. On admission she was found to have low haemoglobin and impaired renal function, deranged LFT and dyselectrolytaemia. Her $\mathrm{Hb}$ was $8.4 \mathrm{gm} / \mathrm{dl}$;

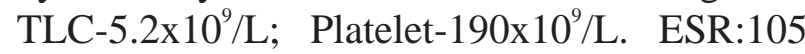
mm (1st Hr.) Creatinine-Serum: 2.15 mg/dl; S. Bilirubin-2.9 mg/dl; ALT (SGPT)-212 iu/L, AST (SGOT)-200 iu/L; Sodium (Na+)-Serum: $143 \mathrm{mmol} / \mathrm{L}$, Potassium (K+)-Serum: 2.6 mmol/L. Complete Hepatitis Profile (Elisa For Hep- A, B, C, E): Negative. Anti dsDNA Antibodies - 56.22 IU/ml. ANA-negative.

She was treated conservatively and received 2 units of blood transfusion. After stabilization upper GI endoscopy was done that revealed haemorrhagic erosion in the body of the stomach. All of a sudden she had severe right hypochondriac pain with regional tenderness which was different from previous abdominal pain. She then consulted with the general surgeon and CT scan of the abdomen was done and found to have a gangrenous and perforated gall bladder and incidentally found to have multiple vascular aneurysms. Subsequently open cholecystectomy was done and biopsy of cystic artery revealed PAN. Her surgical procedure was uneventful. She was consulted with an internist and was treated with IV Methyl prednisolone for 3 days and then oral prednisolone was started. Her biochemical parameter was normalised. She improved clinically and was then discharged with advice and medication.

\section{Discussion}

The vascular lesion in PAN is a necrotizing inflammation of small and medium-sized mus 
cular arteries. The lesions are segmental and tend to involve bifurcations and branching of arteries. They may spread circumferentially to involve adjacent veins. In the acute stages of disease, polymorphonuclear neutrophils infiltrate all layers of the vessel wall and perivascular areas, which results in intimal proliferation and degeneration of the vessel wall. Mononuclear cells infiltrate the area as the lesions progress to the subacute and chronic stages. Fibrinoid necrosis of the vessels ensues with compromise of the lumen, thrombosis, infarction of the tissues supplied by the involved vessel, and, in some cases, hemorrhage. As the lesions heal, there is collagen deposition, which may lead to further occlusion of the vessel lumen. Aneurysmal dilatations up to $1 \mathrm{~cm}$ in size along the involved arteries are characteristic of PAN.

Multiple organ systems are involved, and the clinicopathologic findings reflect the degree and location of vessel involvement and the resulting ischemic changes. The pathology in the kidney in classic PAN is that of arteritis without glomerulonephritis. In patients with significant hypertension, typical pathologic features of glomerulosclerosis may be seen.

Nonspecific signs and symptoms are the hallmarks of PAN. Fever, weight loss, and malaise are present in over one-half of cases. Patients usually present with vague symptoms such as weakness, malaise, headache, abdominal pain, and myalgias that can rapidly progress to a fulminant illness. Specific complaints related to the vascular involvement within a particular organ system may also dominate the presenting clinical picture as well as the entire course of the illness. In PAN, renal involvement most commonly manifests as hypertension, renal insufficiency, or hemorrhage due to microaneurysms. The gastrointestinal manifestations of PAN occur in approximately half of all patients and are among the most challenging symptoms to diagnose correctly because of their nonspecific nature. Postprandial abdominal pain ("intestinal angina") is common. Involvement of the mesenteric arteries in PAN may lead to the disastrous complications of mesenteric infarction or aneurismal rupture, each of which is associated with a high mortality rate. Angiography of the mesenteric vessels reveals multiple microaneurysms. These range in size from lesions that are barely visible to the naked eye to several centimeters in diameter. Sometimes PAN is detected at cholecystectomy or appendectomy in the absence of other disease manifestations. In such cases, surgical removal of the involved organ may be curative.

There are no diagnostic serologic tests for PAN. In $>75 \%$ of patients, the leukocyte count is elevated with a predominance of neutrophils. The anemia of chronic disease may be seen, and an elevated ESR is almost always present. Other common laboratory findings reflect the particular organ involved. Hyper gammaglobulinemia may be present, and up to $30 \%$ of patients have a positive test for hepatitis $B$ surface antigen. Antibodies against myeloperoxidase or proteinase-3 (ANCA) are rarely found in patients with PAN.

The diagnosis of PAN is based on the demonstration of characteristic findings of vasculitis on biopsy material of involved organs. In the absence of easily accessible tissue for biopsy, the angiographic demonstration of involved vessels, particularly in the form of aneurysms of small and medium-sized arteries in the renal, hepatic, and visceral vasculature, is sufficient to make the diagnosis. Aneurysms of vessels are not pathognomonic of PAN. Biopsy of symptomatic organs such as nodular skin lesions, painful testes, and nerve/muscle provides the highest diagnostic yields. 
In patients with idiopathic PAN, glucocorticoids and cytotoxic agents remain the cornerstones of treatment. Approximately half of patients with PAN achieve remissions or cures with high doses of glucocorticoids alone. Cyclophosphamide (eg, $2 \mathrm{mg} / \mathrm{kg} / \mathrm{d}$ orally or $0.6 \mathrm{~g} / \mathrm{m}^{2} / \mathrm{mo}$ intravenously, decreased in the setting of renal dysfunction) is indicated for patients whose disease is refractory to corticosteroids or who have serious involvement of major organs. Favorable therapeutic results have been reported in PAN with the combination of prednisolone and cyclophosphamide. The prognosis of untreated PAN is extremely poor, with a reported 5-year survival rate between 10 and $20 \%$. Death usually results from gastrointestinal complications, particularly bowel infarcts and perforation, and cardiovascular causes. Intractable hypertension often compounds dysfunction in other organ systems, such as the kidneys, heart, and CNS, leading to additional late morbidity and mortality in PAN. In less severe cases of PAN, glucocorticoids alone have resulted in disease remission. Favorable results have also been reported in the treatment of PAN related to hepatitis $\mathrm{B}$ virus with antiviral therapy in combination with glucocorticoids and plasma exchange. Careful attention to the treatment of hypertension can lessen the acute and late morbidity and mortality associated with renal, cardiac, and CNS complications of PAN.

Prophylaxis against Pneumocystis jiroveci (formerly P carinii) pneumonia is an important consideration in patients treated with these medications.

Treatment of HBV-associated PAN with immunosuppressive agents has deleterious long-term effects on the liver. Fortunately, the availability of effective antiviral agents has revolutionized the treatment of HBV-associated cases in recent years. One effective strategy involves the initial use of prednisone (1 $\mathrm{mg} / \mathrm{kg} / \mathrm{d}$ ) to suppress the inflammation. Patients begin 6-week courses of plasma exchange (approximately three exchanges per week) simultaneously with the start of prednisone. The doses of glucocorticoids are tapered rapidly (over approximately 2 weeks), followed by the initiation of antiviral therapy (eg, lamivudine $100 \mathrm{mg} / \mathrm{d})$.

Advanced mononeuritis multiplex can be a severely disabling problem from which recuperation is measured in months or years, if at all. Residual nerve dysfunction in the form of muscle weakness or painful neuropathy is common. The patient's ultimate degree of recovery is difficult to predict. The occurrence of bowel perforation and rupture of a mesenteric microaneurysm are potentially catastrophic events in PAN, requiring emergency surgical intervention and associated with high mortality rates. Patients treated with levels of immunosuppression required for PAN are at substantial risk for opportunistic infection and other complications of treatment.

In contrast to the ANCA-associated vasculitides, which are more prone to recurrences, PAN is generally considered to be a "one-shot" disease. For patients with HBV-associated PAN, seroconversion to anti-HBe antigen antibody usually signals the end of the active phase of vasculitis. Among those with idiopathic PAN, disease recurrences are observed in perhaps $10 \%$ of cases.

\section{References}

1. Fauci, Braunwald, Kasper, Hauser, Longo, Jameson, Loscalzo. Harrison's Principles of Internal Medicine, $17^{\text {th }}$ ed. Part 13. Disorders of the Immune System, Connective Tissue, and Joints. Section 2. Disorders of Immune-Mediated Injury. Chapter 319. The Vasculitis Syndromes.

2. Colmegna I, Maldonado-Cocco JA. Polyarteritis nodosa revisited. Curr Rheumatol Rep. 2005;7:288. [PMID: 16045832]

3. Guillevin L, Mahr A, Callard P. Hepatitis B virus-associated polyarteritis nodosa: clinical characteristics, outcome, and impact of treatment in 115 patients. Medicine (Baltimore) 2005;84:313. [PMID: 16148731] 
4. Stone JH. Polyarteritis nodosa. JAMA. 2002;288:1632. [PMID: 12350194]

5. Achkar AA, Lie JT, Hunder GG, O'Fallon WM, Gabriel SE.et al: How does previous corticosteroid treatment affect the biopsy findings in giant cell (temporal) arteritis? Ann Intern Med. 1994;120:987. [PMID: 8185147]

6. De Groot K, Rasmussen N, Bacon PA. Randomized trial of cyclophosphamide versus methotrexate for induction of remission in early systemic antineutrophil cytoplasmic antibody-associated vasculitis. Arthritis Rheum. 2005;52:2461.

7. Evans JM, O'Fallon WM, Hunder GG. et al. Increased incidence of aortic aneurysm and dissection in giant cell (temporal) arteritis. A population-based study. Ann Intern Med. 1995;122:502. [PMID: 7872584]

8. Fauci AS, Haynes B, Katz P. The spectrum of vasculitis: clinical, pathologic, immunologic, and therapeutic considerations. Ann Intern Med. 1978;89:660. [PMID: 31121]

9. Guillevin L, Lhote F, Sauvaget F, et al. Treatment of polyarteritis nodosa related to hepatitis B virus with interferon-alpha and plasma exchanges. Ann Rheum Dis. 1994;53:334. [PMID: 7912504]

10. Guillevin L. Churg-Strauss syndrome. Clinical study and long-term follow-up of 96 patients. Medicine (Baltimore). 1999;78:26. [PMID: 9990352]

11. Hoffman GS, Leavitt RY, Kerr GS, et al: Treatment of glucocorticoid-resistant or relapsing Takayasu arteritis with methotrexate. Arthritis Rheum. 1994;37:578. [PMID: 7908520]

12. Hoffman GS, Cid MC, Hellmann DB, et al. A multicenter, randomized, double-blind, placebo-controlled trial of adjuvant methotrexate treatment for giant cell arteritis. Arthritis Rheum. 2002;46:1309. [PMID: 12115238]

13. Jayne D, Rasmussen N, Andrassy K, et al. A randomized trial of maintenance therapy for vasculitis associated with antineutrophil cytoplasmic autoantibodies. N Engl J Med. 2003;349:36. [PMID: 12840090]
14. Merkel PA. Drug-induced vasculitis. Rheum Dis Clin North Am. 2001;27:849. [PMID: 11723768]

15. Hoffman GS, Cid MC, Hellmann DB. Brief communication: High incidence of venous thrombotic events among patients with wegener granulomatosis. The wegener's clinical occurrence of thrombosis (WeCLOT) study. Ann Intern Med. 2005;142:620. [PMID: 15838068]

16. Hoffman GS, Cid MC, Hellmann DB, et al. Low-dose aspirin and prevention of cranial ischemic complications in giant cell arteritis. Arthritis Rheum. 2004;50:1332. [PMID: 15077317]

17. Salvarani C, Cantini F, Boiardi L, et al: Polymyalgia rheumatica and giant-cell arteritis. N Engl J. Med. 2002;347:261. [PMID: 12140303]

18. Sneller MC, Fauci AS. An analysis of forty-two wegener's granulomatosis patients treated with methotrexate and prednisone. Arthritis Rheum. 1995;38:608. [PMID: 7748215]

19. Talar-Williams C, Hijazi YM, Walther MM. Cyclophosphamide-induced cystitis and bladder cancer in patients with Wegener's granulomatosis. Ann Intern Med. 1996;124:477. [PMID: 8602705]

20. Vassilopoulos D, Calabrese LH. Hepatitis C virus infection and vasculitis: implications of antiviral and immunosuppressive therapies. Arthritis Rheum. 2002;46:585. [PMID: 11920393]

21. WGET Research Group: Etanercept plus standard therapy for wegener's granulomatosis. N Engl J Med. 2005;352:351.

22. Weyand CM, Goronzy JJ: Medium- and large-vessel vasculitis. $\mathrm{N}$ Engl $\mathrm{J}$ Med. 2003;349:160. [PMID: 12853590]

23. Weyand CM, Goronzy JJ: Giant-cell arteritis and polymyalgia rheumatica. Ann Intern Med. 2003;139:505. [PMID: 13679329]

24. Xiao H, Heeringa P, Hu P. Antineutrophil cytoplasmic autoantibodies specific for myeloperoxidase cause glomerulonephritis and vasculitis in mice. J Clin Invest. 2002;110:955. [PMID: 12370273] 